\title{
Micromachined Fabry-Perot Optical Filters
}

\author{
M. Bartek, J. H. Correia and R. F. Wolffenbuttel \\ Delft University of Technology, ITS/Et, Laboratory for Electronic \\ Instrumentation/DIMES, Mekelweg 4, 2628 CD Delft, The Netherlands \\ e-mail: M.Bartek@its.tudelft.nl, J.H.Correia@its.tudelft.nl and \\ R.F.Wolffenbuttel@its.tudelft.nl
}

\begin{abstract}
The design, fabrication and measured characteristics of micromachined FabryPerot (F-P) optical filters for the visible spectral range are presented. Silver films of 40-50 nm thickness, evaporated on a $300 \mathrm{~nm}$ thick low-stress silicon nitride membrane, are used as high-quality mirrors. Two parallel mirrors, with a square aperture of up to $2 \times 2 \mathrm{~mm}^{2}$ and initial cavity gap of $1.2 \mu \mathrm{m}$, form a tunable FabryPerot optical filter. One of the mirrors is fixed the other is under tension on a movable Si frame, which is electrostatically deflected to control the mirror spacing and parallelism. Results are compared with non-tunable F-P filters that are composed of an $\mathrm{Ag} / \mathrm{SiN} / \mathrm{Ag}$ or $\mathrm{Ag} / \mathrm{SiO}_{2} / \mathrm{Al}$ layer stack. The FWHM of $40 \mathrm{~nm}$ (tunable filter) and $16 \mathrm{~nm}$ (non-tunable filter) have been achieved.
\end{abstract}

\section{Introduction}

In recent years much attention has been paid to the development of tunable micromachined optical filters based on a Fabry-Perot (F-P) resonator (Fig. 1) [1, 2, 3]. Research has been primarily focused on the near-infrared region (wavelength of 1.3 and $1.55 \mu \mathrm{m})$ because of interest in the multimode optical fibre communication. Attempts to fabricate a device in the visible spectral

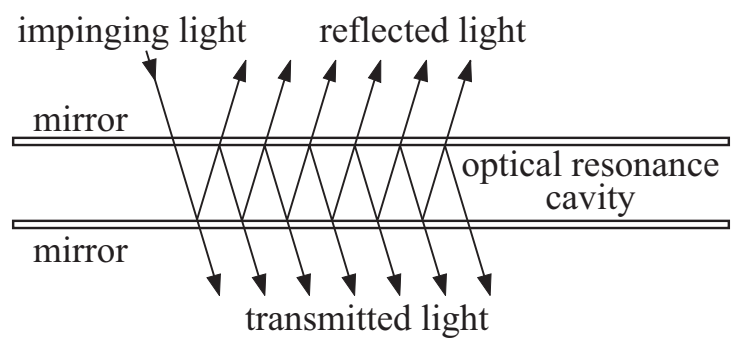

Fig. 1: Fabry-Perot optical resonator. range have also been reported [4], but were less successful. The goal is to develop an integrated spectrometer. In such an application, the requirements are much more demanding (wide optical band operation, the mirror flatness is more critical). This paper presents design, fabrication and characterisation of a miniature F-P interferometer based on silver mirrors for the visible spectral range.

\section{Micromachined Fabry-Perot optical filters}

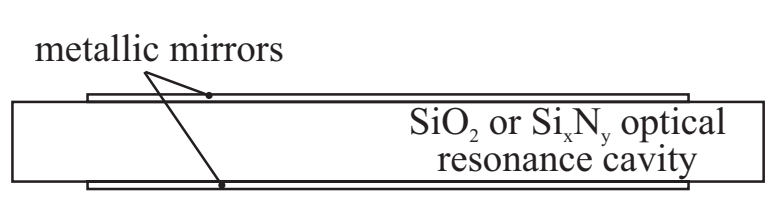

(a)

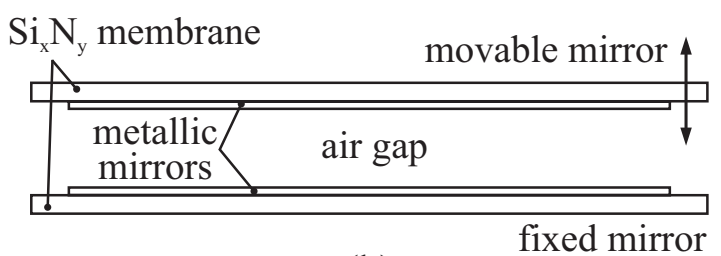

(b)

Fig. 2: Non-tunable (a) and tunable (b) F-P optical filters have been investigated.

Two types of F-P filters have been investigated: non-tunable and tunable (see Fig. 2). In the non-tunable filter the mirror spacing is fixed and is equal to the layer thickness deposited 
during fabrication. In the tunable filter, one of the mirrors is movable, resulting in adjustable mirror spacing.

input radiation

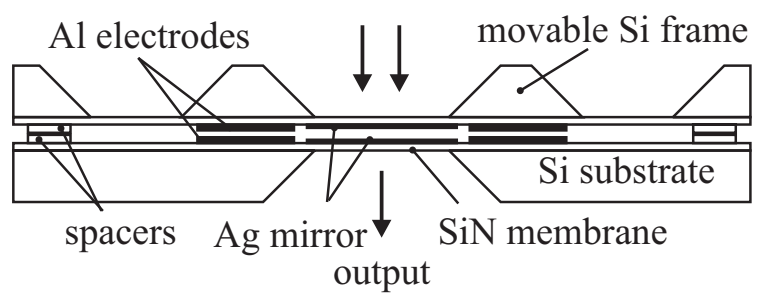

Fig. 3: Cross-section of the proposed micromachined F-P optical filter.
The proposed structure of the tunable filter is shown schematically in cross-section in Fig. 3. The device is formed by two parallel $40 \mathrm{~nm}$ thick silver mirrors supported by a $300 \mathrm{~nm}$ low-stress silicon nitride membrane. The mirrors have a square aperture of $2 \times 2 \mathrm{~mm}^{2}$ and an initial cavity gap of $1.2 \mu \mathrm{m}$. One of the mirror is fixed, the other is under tension on a movable $\mathrm{Si}$ frame, which is electrostatically deflected using several distributed electrodes to control the mirror spacing and parallelism.

A thin film optics software package (TFCalc 3.2.5) was used to perform optimisation of the mirror layer thickness, composition and order (optical data [5, 6]). The silver layers must be inside the resonance cavity to avoid excessive absorption losses in the silicon nitride layer during multiple reflections. The Ag layer thickness is a trade off between achievable FWHM (Full-Width-Half-Maximum) and absorption loss. Silver was selected as the mirror material because of its high reflectivity $(>90 \%)$ over the entire visible spectral range and fabrication simplicity [7]. The poor long-term stability [8] of silver-based reflective coatings in macroscopic applications can be avoided in microsystems, as the dimensions allow protection by sealing.

Geometrical form, bending distances, deflection, stress, fatigue and flatness of the diaphragm based on a low stress silicon nitride membrane/Si frame were simulated in order to
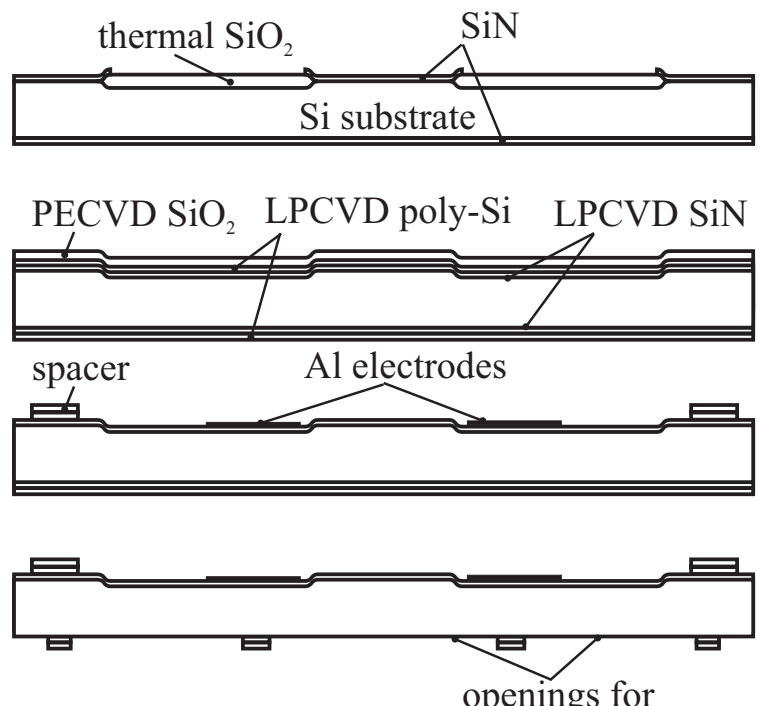

Ag mirror anisotropic $\mathrm{KOH}$ etching
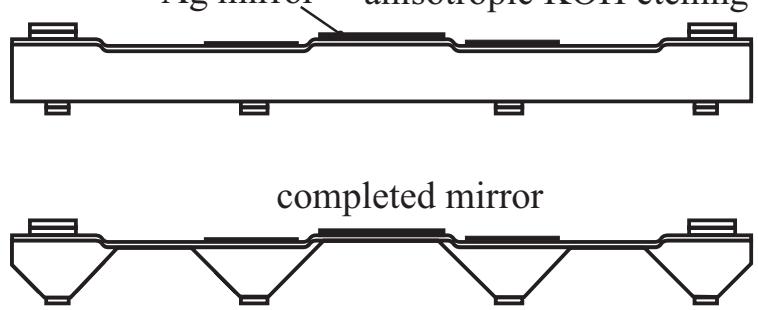

Fig. 4: Schematic fabrication sequence. study the mechanical behaviour of a movable mirror. The results of FEM simulations predict excellent flatness of the movable mirror in the whole range of required deflections $(0-460 \mathrm{~nm}$ for control voltages of 0-21 V) and have been published previously [9].

\section{Fabrication}

On the same wafer $(100 \mathrm{~mm}$, double-side polished), upper and bottom dies have been fabricated using a 5-mask process (see Fig. 4). Firstly, $400 \mathrm{~nm}$ recesses are formed using LOCOS. Subsequently, a $300 \mathrm{~nm}$ low-stress $(<0.15 \mathrm{GPa})$ LPCVD silicon nitride layer is deposited and protected by a $300 \mathrm{~nm}$ LPCVD poly-Si layer. Then, PECVD oxide $(0.3-1 \mu \mathrm{m})$ is deposited on a wafer front-side with thickness corresponding to the required initial resonance cavity gap. The PECVD-oxide/poly$\mathrm{Si}$ stack is patterned to form spacers between upper and bottom dies for later die attachment. The $300 \mathrm{~nm} \quad \mathrm{Al}$ interconnect and control/sensing electrodes (deposited by sputtering) are 'buried' in $400 \mathrm{~nm}$ recesses to 
increase the initial spacing of the electrodes and avoid sticking during operation. The wafer backside is patterned to prepare windows for anisotropic $\mathrm{KOH}$ etching. Silver mirror layers are evaporated and patterned using lift-off on the wafer front side. The anisotropic $\mathrm{KOH}$ etching (33 wt.\% $\mathrm{KOH}$ solution at $85^{\circ} \mathrm{C}$ ) is performed in a holder to protect the Ag mirrors. To facilitate the wafer dicing into the individual dies, deep V-shaped trenches are formed during $\mathrm{KOH}$ etching. After the bottom die is mounted on a PCB, the upper die is attached using glue. Fig. 5 shows a photograph of the fabricated

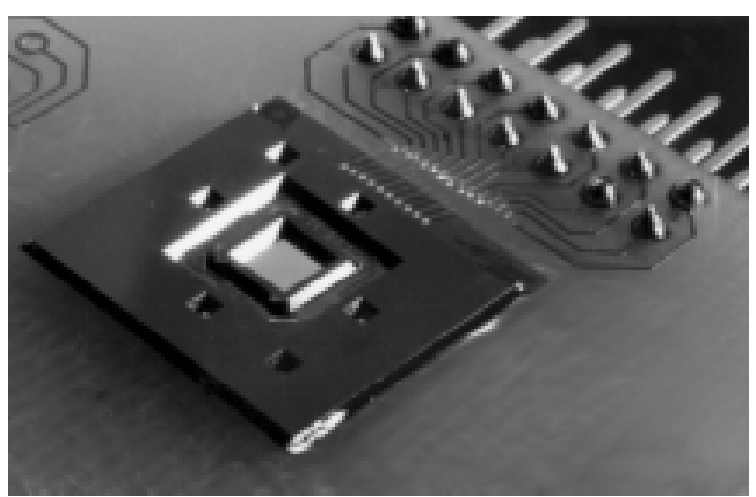

Fig. 5: A photograph of the fabricated miniature tunable F-P optical filter. device.

\section{Results}

To test the concept of silver-based mirrors, a $40 \mathrm{~nm} \mathrm{Ag}$ layer was deposited on a SiN membranes from both sides resulting in a non-tunable F-P filter. The interference measurements (projected interference fringes) have shown an excellent mirror flatness. The LPCVD silicon nitride membrane, which is under tension after the release using anisotropic $\mathrm{KOH}$ etching, improves the initial flatness of the silver mirror.
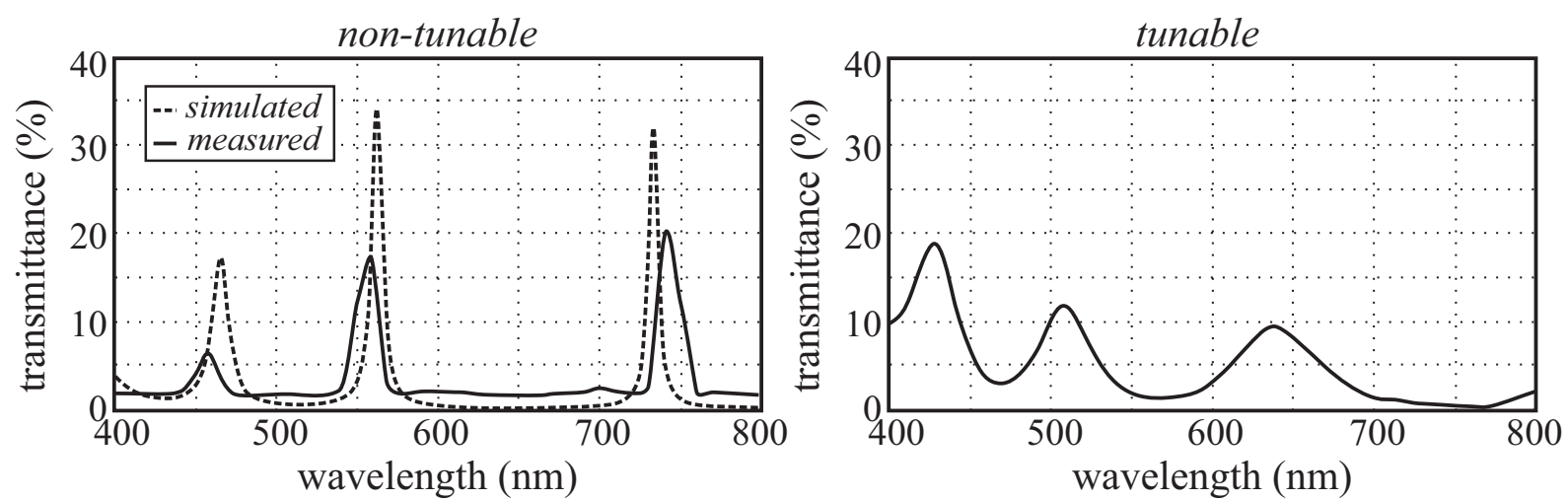

Fig. 6: Transmittance vs. wavelength for a $40 \mathrm{~nm}-\mathrm{Ag} / 450 \mathrm{~nm}-\mathrm{SiN} / 40 \mathrm{~nm}-\mathrm{Ag}$ fixed layer stack (at the left); and a 300nm-SiN/40nm-Ag/1000nm-air/40nm-Ag/300nm-SiN tunable layer stack (at the right).

The spectral characteristics in transmittance have been measured between $400 \mathrm{~nm}$ to $800 \mathrm{~nm}$ using Oriel 77250 monochromator. Fig. 6 compares the results achieved for both nontunable and tunable F-P filters. At the left, the simulated and measured transmittance for a 40nm-Ag/450nm-SiN/40nm-Ag non-tunable F-P filter is shown. The FWHM of $20 \mathrm{~nm}$ is in good agreement with simulations. The tunable filter (Fig. 6, at the right) shows the FWHM of $40 \mathrm{~nm}$ which is much more less than the predicted value ( $8 \mathrm{~nm}$ for air gap of $\sim 500 \mathrm{~nm}$ ). This is caused probably due to mirror non-parallelism. It was found that it is difficult to achieve acceptable mirror parallelism by manual adjustment of the voltages applied on the control electrodes.

The best results have been achieved for an F-P filter composed of a $45 \mathrm{~nm}-\mathrm{Ag} / 300 \mathrm{~nm}$ $\mathrm{SiO}_{2} / 20 \mathrm{~nm}-\mathrm{Al}$ layer stack (see Fig. 7). A single peak with the FWHM of $16 \mathrm{~nm}$ over the 
entire visible spectral range was achieved. This type of filter is suitable for an array-type micro-spectrometer.

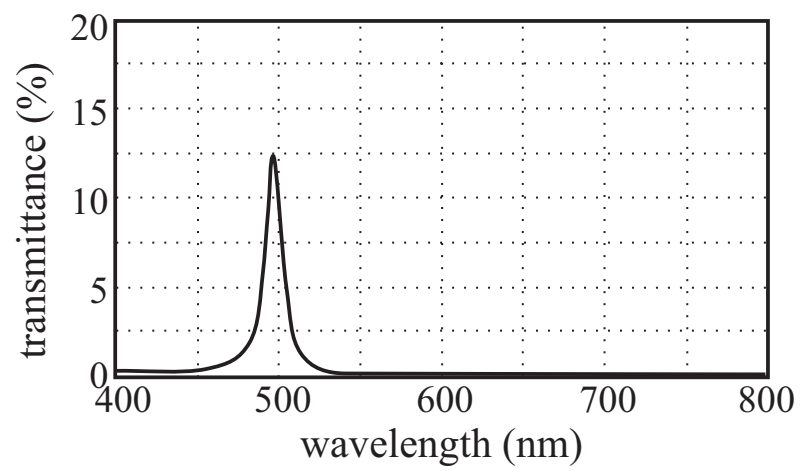

Fig. 7: Measured transmittance vs. wavelength for a $45 \mathrm{~nm}-\mathrm{Ag} / 300 \mathrm{~nm}-\mathrm{SiO}_{2} / 20 \mathrm{~nm}-\mathrm{Al}$ filter.

\section{Conclusions}

The design, fabrication and measured characteristics of micromachined Fabry-Perot optical filters for the visible spectral range are presented. Silver films of 40-50 nm thickness, evaporated on a $300 \mathrm{~nm}$ thick low-stress silicon nitride membrane, are used as high-quality mirrors. Two parallel mirrors, with a square aperture of up to $2 \times 2 \mathrm{~mm}^{2}$ and initial cavity gap of $1.2 \mu \mathrm{m}$, form a tunable Fabry-Perot optical filter. One of the mirrors is fixed the other is under tension on a movable Si frame, which is electrostatically deflected to control the mirror spacing and parallelism. Results are compared with non-tunable F-P filters that are composed of an $\mathrm{Ag} / \mathrm{SiN} / \mathrm{Ag}$ or $\mathrm{Ag} / \mathrm{SiO}_{2} / \mathrm{Al}$ layer stack. The FWHM of $40 \mathrm{~nm}$ (tunable filter) and $16 \mathrm{~nm}$ (non-tunable filter) have been achieved.

\section{Acknowledgement}

The authors wish to thank the staff of Delft Institute of Microelectronics and Submicron Technology (DIMES), especially J. Groeneweg, for technical assistance in fabrication of the devices. The work presented here has been supported in part by STW, project DEL 55.3733, and JNICT - Portugal, PRAXIS XXI-BD/5181/95.

\section{References}

[1] A.T.T.D. Tran, et al., IEEE Photonics Technology Lett. 8, 3, 1996, pp. 393-395.

[2] G.L. Christenson, A.T.T. Tran, Z.H. Zhu, Y.H. Lo, in Proc. MEMS '97, Nagoya, Japan, 1997, pp. 61-65.

[3] J. Patterson, B. van Zeghbroeck, in Digest IEEE/LEOS 1996 Summer topical meeting on optical MEMS and their appl., 1996, pp. 25-26.

[4] N.F. Raley, et al., in Proc. 5th IEEE Solid State Sensor and Actuator Worksh., Hilton Head Island, SC, USA, 1992, pp. 170-173.

[5] D.P. Poenar, R.F. Wolffenbuttel, Applied Optics 36, 21, July 1997, pp. 5122-5128.

[6] H.A. Macleod, Thin-film optical filters, Adam Hilger Ltd, 2nd edition, 1986.

[7] M. Bartek, J.H. Correia, and R.F. Wolffenbuttel, in Proc. MME '98, Ulvik, Norway, 1998, p. 43-46.

[8] D.-Y. Song, R. W. Sprague, H. A. Macleod, M. Jacobson, Appl. Optics 24, 8, 1985, pp. 1164-1170.

[9] J.H. Correia, M. Bartek and R.F. Wolffenbuttel, in Proc. Modeling and Simulations of Microsystems '98, Santa Clara, CA, USA, 1998, p. M3.5.5. 